Edición Extraordinaria. p.p.1019-1032

Memorias del VIII Encuentro Nacional de Experiencias en Enseñanza de la Biología y la Educación Ambiental. III Congreso Nacional de Investigación en Enseñanza de la Biología.

\title{
CONCEPCIONES SOBRE TRABAJOS DE CAMPO EN LA ENSEÑANZA DE LA BIOLOGÍA DE FUTUROS DOCENTES DE COLOMBIA, ESPAÑA Y ARGENTINA
}

\section{CONCEPTS ON FIELD WORK IN THE TEACHING OF BIOLOGY OF FUTURE TEACHERS OF COLOMBIA, SPAIN AND ARGENTINA}

\author{
Nancy Fernández ${ }^{1}$ \\ Emilio Costillo Borrego ${ }^{2}$ \\ Elías Francisco Amórtegui Cedeño ${ }^{3}$
}

\section{RESUMEN}

En este documento presentamos resultados preliminares acerca del estudio de las concepciones de futuros maestros de ciencias naturales que cursan espacios académicos de Didáctica en tres universidades: Universidad Surcolombiana, Universidad de Extremadura y del Instituto de Formación Docente de Ushuaia. El estudio se llevó a cabo desde una perspectiva cualitativa, con base en el análisis de contenido y empleando como técnica de recolección de información un cuestionario validado por expertos; las categorías de estudio corresponden al análisis del trabajo práctico de campo en la enseñanza de la Biología. Los resultados muestran la predominancia en algunos casos de dichas concepciones en modelos tradicionales de enseñanza, sin embargo, destacamos la importancia del inicio de sistemas de ideas más complejos y de enfoque más constructivista hacia la enseñanza de la Biología de los futuros docentes lberoamericanos.

\section{SUMMARY}

In this paper we present preliminary results on the study of the conceptions of future teachers of natural sciences who attend academic spaces of Teaching at three universities: Surcolombiana University, University of Extremadura and the Teacher Training Institute of Ushuaia. The study was conducted from a qualitative perspective, based on content analysis and using such information collection technique questionnaire validated by experts; categories of study for the analysis of practical fieldwork in teaching biology. The results show the predominance in some cases these concepts in traditional teaching models, however, stress the importance of starting systems more complex ideas and toward more constructivist teaching Biology ibero teachers future cohort.

PALABRAS CLAVE: Trabajo Práctico, Práctica de Campo, Concepciones.

KEYWORDS: Practical work, Field Practice, Conception.

Universidad Nacional de Tierra del Fuego, Argentina. nfernandez@untdf.edu.ar

Universidad de Extremadura, España. emiliocostillo@gmail.com

Universidad Surcolombiana, Colombia. elias.amortegui@usco.edu.co 
Edición Extraordinaria. p.p.1019-1032

Memorias del VIII Encuentro Nacional de Experiencias en Enseñanza de la Biología y la Educación Ambiental. III Congreso Nacional de Investigación en Enseñanza de la Biología.

\section{INTRODUCCIÓN}

Desde la perspectiva del Conocimiento Profesional del Profesor, las Prácticas de Campo y en general, los Trabajos Prácticos, son considerados estrategias de enseñanza, y desde esta perspectiva son un componente fundamental en el Conocimiento Didáctico del Contenido (Valbuena, 2007), sin embargo las investigaciones sobre el Conocimiento Profesional de Profesores de Ciencias Naturales y Educación Ambiental son escasos y en su mayoría se han realizado respecto al conocimiento disciplinar que se enseña en cada una de las disciplinas, sean Física, Química o Biología (Subject matter Knowledge).

El Trabajo Práctico y la formación del profesorado en la enseñanza de las Ciencias han venido constituyéndose en una faceta muy valorada por los investigadores (Rodrigo et al, 1999; Caamaño, 2003). Específicamente las Prácticas de Campo (PC) se consideran importantes en la formación inicial de profesores de Ciencias (Dourado, 2006), al concebirse como una estrategia de enseñanza que permite relacionar la teoría con la práctica y favorecer el aprendizaje de diversos contenidos, constituyendo además una fuente de información directa, ejemplos y experiencias.

La revisión de antecedentes muestra que existe una gran cantidad de investigaciones sobre los Trabajos Prácticos en Ciencias, en mayor medida a partir de revisiones bibliográficas. Otro ámbito en el que se han centrado las investigaciones sobre los Trabajos Prácticos, ha sido particularmente las Prácticas de Laboratorio en secundaria y en educación superior con relación a concepciones de estudiantes, profesores y futuros profesores. Las investigaciones sobre las Prácticas de Campo son escasas (Amórtegui 2011; Amórtegui y Correa 2012).

En algunos casos, las investigaciones corresponden a las concepciones de profesores de ciencias naturales en educación primaria y secundaria, tanto a nivel anglosajón como latinoamericano. En una mayor proporción, las investigaciones sobre las Prácticas de Campo se han desarrollado en el área de la Geología, algunas corresponden a la implementación de estrategias de campo y enseñanza de esta disciplina y otras corresponden a la formación de profesores; inclusive se encuentran investigaciones relacionadas con la enseñanza de la Geografía. Por último, los estudios sobre Trabajos Prácticos y formación del profesorado de ciencias naturales son pocos, se destacan los trabajos con profesores de ciencias naturales en secundaria. Estos últimos han abordado las concepciones e ideas de los profesores en ejercicio durante sus clases de ciencias (Berezuki, Obara y Silva, 2009).

Tan solo dos investigaciones han abordado la relación y el aporte de las Prácticas de Campo en la construcción del Conocimiento Profesional de profesores en formación inicial (Morcillo et al, 1999; Amórtegui y Correa, 2012) Lo anterior muestra el vacío que existe en este tipo de investigaciones, lo que genera la importancia de analizar el papel de las Prácticas de Campo en los programas curriculares de formación de docentes en Ciencias.

Esta revisión de antecedentes que han posibilitado construir un estado del arte, muestra que en términos generales los estudios sobre prácticas de campo se han centrado en cuatro tendencias. La primera corresponde a reflexiones o revisiones sobre dichas actividades, particularmente desde la perspectiva documental y en el contexto anglosajón. 
Edición Extraordinaria. p.p.1019-1032

Memorias del VIII Encuentro Nacional de Experiencias en Enseñanza de la Biología y la Educación Ambiental. III Congreso Nacional de Investigación en Enseñanza de la Biología.

La segunda tendencia corresponde a investigaciones cuyo objeto de estudio han sido la relación entre las prácticas de campo y la enseñanza de las ciencias naturales en general. Aquí tan solo se halla un trabajo que corresponde al diseño de una salida de campo (Alarcón y Piñeros, 1989).

La tercera tendencia corresponde a las concepciones sobre prácticas de campo en la enseñanza de las ciencias. Allí los trabajos han sido mayoritariamente desde una perspectiva cualitativa en donde ha predominado el uso de instrumentos como el cuestionario. Esta tendencia se ha visto marcada por su población objeto de estudio, pues en su mayoría ha correspondido a maestros en ejercicio en los niveles primarios y secundarios.

Por último, la cuarta tendencia corresponde a las investigaciones sobre prácticas de campo y su relación con el pensamiento-conocimiento del profesor. Tan solo dos investigaciones se han centrado en el contexto de la formación inicial de profesores de Biología y el aporte de las prácticas de campo a la construcción del Conocimiento Profesional del Profesor (Amórtegui, Gutiérrez y Medellín, 2009; Amórtegui y Correa, 2009). Sin embargo estas investigaciones no se han centrado en los procesos formativos y en las concepciones de los futuros maestros sobre dichas actividades.

En esta ponencia nos permitimos abordar las concepciones de futuros profesores de ciencias naturales sobre trabajos de campo en la enseñanza de las ciencias, en tres instituciones de Educación Superior, Instituto de Formación de docentes de Tierra de Fuego en Argentina, Universidad de Extremadura en España y Universidad Surcolombiana en Colombia.

\section{METODOLOGÍA}

El estudio se llevó a cabo desde un enfoque cualitativo, empleando el método de análisis de contenido en el proceso de sistematización, y usando como herramienta de recolección de información un cuestionario validado y empleado en estudios anteriores por Amórtegui (2011). En la población nos referimos a tres grupos de trabajo: el primero corresponde a 13 estudiantes entre 19 y 25 años de edad, pertenecientes en su mayoría a los estratos socioeconómicos 1, 2 y 3 y que representan la totalidad de asistentes del espacio académico ofrecido en sexto semestre en el Programa de Licenciatura en Ciencias Naturales: Física, Química, Biología de la Universidad Surcolombiana-USCO (Neiva, Colombia); el segundo consistió en un grupo de 15 profesores de Primaria en formación que cursan su tercer año en el Grado en Educación Primaria de la Universidad de Extremadura (Badajoz, España).

El tercer grupo, corresponde a 7 estudiantes entre 20 y 27 años, cursantes del cuarto año del espacio Didáctica Especial III del Profesorado de Biología del Instituto de Formación Docente de la Ciudad de Ushuaia (Tierra del Fuego, Argentina)

Para la sistematización y análisis de los resultados tuvimos en cuenta las categorías propuestas por Puentes y Valbuena (2011) y enriquecidas por Amórtegui y Correa (2012), estas se muestran en la Tabla 1. 
Edición Extraordinaria. p.p.1019-1032

Memorias del VIII Encuentro Nacional de Experiencias en Enseñanza de la Biología y la Educación Ambiental. III Congreso Nacional de Investigación en Enseñanza de la Biología.

Tabla 1. Categorías de la investigación (Tomado de Amórtegui y Correa, 2012; Puentes y Valbuena, 2011).

\begin{tabular}{|c|c|}
\hline Categoría & Subcategorías \\
\hline \multirow[t]{3}{*}{ NATURALEZA } & Imagen de práctica \\
\hline & Relación teoría- práctica \\
\hline & Clase de Trabajo Práctico \\
\hline \multicolumn{2}{|l|}{ FINALIDADES } \\
\hline \multirow[t]{4}{*}{ METODOLOGÍA } & Estructura de la Guía de Campo \\
\hline & Enfoque didáctico \\
\hline & Momento de realización \\
\hline & Roles \\
\hline \multirow[t]{3}{*}{ EVALUACIÓN } & Tipos \\
\hline & Roles \\
\hline & Contenidos a evaluar \\
\hline \multirow{4}{*}{$\begin{array}{l}\text { RELACIÓN } \quad \text { CON } \\
\text { EPISTEMOLÓGICOS }\end{array}$} & Biología como Ciencia \\
\hline & Objeto de estudio de la Biología \\
\hline & Producción de conocimiento biológico \\
\hline & Imagen de Ciencia \\
\hline \multicolumn{2}{|l|}{$\begin{array}{l}\text { ASPECTOS RELACIONADOS CON LA } \\
\text { FORMACIÓN DOCENTE }\end{array}$} \\
\hline $\begin{array}{l}\text { ASPECTOS RELACIONADOS CON EL } \\
\text { CURRÍCULO DEL PCLB }\end{array}$ & \\
\hline
\end{tabular}

\section{RESULTADOS}

A continuación presentamos los hallazgos para cuatro sub categorías de investigación. En algunos casos ilustramos con declaraciones textuales de los futuros profesores (se destaca la información relevante subrayando) y nos referimos a cada grupo de estudio particular para posteriormente hacer un análisis general.

\section{El caso colombiano}

Con relación a la Imagen de Práctica de Campo, los futuros docentes del Departamento del Huila identifican tres tendencias: como vivencia (6 sujetos), como actividad (4 sujetos) y como estrategia de enseñanza (3 sujetos).

Frente a la primera se refieren a la salida de campo como una vivencia que no se instala específicamente en el marco de la enseñanza-aprendizaje de las ciencias, sino que permite desarrollar ciertos tipos de actividades de características experimentales.

E8. "Es una vivencia en la cual podemos aplicar todo lo aprendido en el aula de clases, donde podemos aplicar todos los métodos y maneras de asumir cuestiones a través de la toma de datos y recolección de muestras..." 
Memorias del VIII Encuentro Nacional de Experiencias en Enseñanza de la Biología y la Educación Ambiental. III Congreso Nacional de Investigación en Enseñanza de la Biología.

A diferencia de lo anterior, un pequeño grupo de estudiantes afirma la importancia de la práctica de campo como una estrategia de enseñanza de la Biología.

E4. "Una salida de campo es una estrategia metodológica de enseñanza donde se requiere salir de las aulas de clase para llevar a cabo el desarrollo de actividades con el medio de manera más práctica...".

Frente a la Relación teoría-práctica existe una marcada tendencia (10) a considerar esta estrategia como la aplicación, contrastación, verificación de la teoría.

E2. "...la salida de campo se realiza con el fin de que el estudiante vea claramente lo que se le ha explicado en clase..."

Destacamos que tan solo un futuro docente concibe la posibilidad de que la práctica de campo sea un espacio de construcción de conocimientos.

E12. "Hay bastante relación pero también hay que tener en cuenta que durante una salida de campo también se aprenden cosas nuevas, experiencias, costumbres, métodos, culturas sobre algún tema determinado...”.

Por último para este primer caso, las finalidades muestran dos tendencias. En la primera, 10 futuros docentes explicitan que la salida de campo se realiza principalmente para corroborar la teoría y los temas vistos en clase, así como experimentar y tomar muestras; de manera minoritaria tres futuros docentes contemplan además de aspectos conceptuales, elementos actitudinales tales como la generación de conciencia sobre las problemáticas ambientales.

E10. "Una salida de campo puede estar enfatizada en un reconocimiento de la riqueza, la concienciación sobre éstos mismos, explicación de los temas...".

\section{El caso argentino}

Con relación a la Imagen de Práctica de Campo, los futuros docentes de Ushuaia, identifican tres tendencias: como lugar donde encontrar información (2 sujetos), como aplicación de la teoría (2 sujetos) y como estrategia de enseñanza (3 sujetos).

Con respecto a la primera imagen, se refieren a la salida de campo como un lugar donde encontrar información que puede utilizarse luego en las clases teóricas, no se indica que tuviera relación con la enseñanza de las ciencias, sino que permitiría desarrollar ciertos tipos de actividades de características experimentales.

E3U: “Es ir al lugar donde ha ocurrido u ocurre lo que quiero estudiar, para poder observar y registrar y aprender en el contexto"

En relación a la segunda concepción, existe una similar tendencia (2) a considerar esta estrategia como la aplicación, contrastación, verificación de la teoría. 
Memorias del VIII Encuentro Nacional de Experiencias en Enseñanza de la Biología y la Educación Ambiental. III Congreso Nacional de Investigación en Enseñanza de la Biología.

E4U: "Salida de campo, para mi es cuando se aprende algo ya conocido anteriormente en la teorís, pero de maera concreta, tangible y no abstracta como lo es muchas veces el aula"

El resto de las estudiantes (3), plantea la importancia de la práctica de campo como una estrategia de enseñanza de la Biología.

E2U: "se refiere a una salida en el ámbito educativo, liderada por un docente, con un fin especificado previamente; en dicha salida se deberán recoger datos , observaciones e información que será orientada por el docente. Y luego se reforzará con contenidos teóricos en las clases habituales

En este caso, las estudiantes mencionadas, plantean que los TPC, se realizan en el marco de una actividad educativa.

Al respecto de la finalidad de los TPC, podemos identificar cuatro (4) tipos de concepciones. Tres (3) de ellas se refieren a poder establecer relaciones entre lo aprendido en la teoría y la práctica de campo. Lo plantean como una relación dinámica de ida y vuelta, en la cual ambas actividades teoría y práctica se retroalimentan entre sí.

E5U: "Las finalidades son obtener ejemplos y realizar relaciones sumado lo teórico y así comprender mejor el /los temas trabajados"

Una de las estudiantes menciona que la finalidad es aplicar la teoría

E1U: "La finalidad de una salida de campo es observar los contenidos trabajados en su estado natural, para poder interpretar la relación que tiene con todo el ambiente físico."

Una (1) plantea que la finalidad es promover estrategias de recolección de datos.

E2U: "Creo que la finalidad es acercar a los alumnos a los fenómenos naturales, obtener datos, observaciones".

Y sólo una (1) de las estudiantes encuestadas, plantea aspectos vinculados a lo vivencial $y$ emocional de una actividad de campo.

E3U: "Uno vivencia desde otro lugar el aprendizaje con la salida de campo, los contenidos ya no se olvidan o podemos recordarlos más rápido a través de traer a la memoria aquella experiencia. Se vive $y$ además es inevitablemente es interdisciplinaria"

En el caso Argentino, es posible identificar una gran variedad de concepciones sobre los | Trabajos prácticos de campo. 
Edición Extraordinaria. p.p.1019-1032

Memorias del VIII Encuentro Nacional de Experiencias en Enseñanza de la Biología y la Educación Ambiental. III Congreso Nacional de Investigación en Enseñanza de la Biología.

\section{El caso español}

En los futuros docentes de la Universidad de Extremadura también se pueden identificar tres tendencias con respecto a la Imagen de Práctica de Campo. Una parte importante de estos futuros docentes (7) la entiende como actividades prácticas de carácter científico-escolar.

E3E. "Es una manera diferente de dar clase, donde además de los propios contenidos también educamos valores diferentes a los del aula. Es un apoyo didáctico diferente a lo que estamos acostumbrados, es un estilo de escuela diferente, más participativa...".

Es destacable el número de profesores en formación (5) que señalan el carácter de vivencia de estas actividades. Por último, 3 futuros docente indican la relación existente en estas actividades entre la teoría y la práctica como estrategias de enseñanza.

En cuanto a la Relación Teoría-Práctica la mayoría de los profesores en formación (12) considera que en las Prácticas de Campo se comprueba, relaciona, corrobora...la teoría del aula con la práctica del medio natural. No obstante de estos profesores dos señalan que además estas actividades permiten el aprendizaje significativo de los alumnos.

E4E. “La relación más básica consiste en encontrar un sentido práctico y utilitario a lo estudiado. También es posible que mediante dicha actividad entendamos conceptos que antes no comprendíamos...".

En menor medida, unos pocos futuros docentes (3) integran estas actividades junto con la teoría en el proceso de enseñanza de los alumnos.

E11E. "Con los temas tratados en clase podemos realizar esta tipo de salidas para estudiar las especies, ecosistemas, etc de una manera más directa...".

Con respecto a la finalidad, los profesores en formación señalan distintos fines de las Prácticas de Campo; 4 de ellos incluso en su respuesta reflejan que los objetivos pueden ser múltiples. Para muchos de ellos (9) la finalidad de estas actividades está relacionada con mejoras en el proceso de enseñanza-aprendizaje de las Ciencias Experimentales.

E1E. "La finalidad principal de realizar una salida de campo yo creo que es que los niños no vean la asignatura como algo teórico que no existen en la práctica, en la vida cotidiana, sino que todo lo estudiado lo podemos observar y comprender de una manera práctica...".

En 6 ocasiones se señala que la comprobación, hacer realidad la teoría,... es una de las finalidades de las Prácticas de Campo.

E12E. "Comprobar lo estudiado en el aula y que los alumnos puedan ver con sus propios ojos la teoría para que la recuerden mejor". 
Edición Extraordinaria. p.p.1019-1032

Memorias del VIII Encuentro Nacional de Experiencias en Enseñanza de la Biología y la Educación Ambiental. III Congreso Nacional de Investigación en Enseñanza de la Biología.

En 3 ocasiones se mencionan aspectos relacionados con vivencias como un objetivo de estas prácticas que pueden ayudar a aumentar el interés de los alumnos por la ciencia.

E2E. “...Creo que también sirve de relajación y de estimulación...”.

En un solo caso, un futuro docente estableció la educación ambiental como un objetivo de las Prácticas de Campo.

Por último, a manera de análisis quisiéramos destacar que en algunos casos la Imagen de Práctica y la Relación Teoría-Prática, se asocian teóricamente al modelo de Transmisión recepción planteado por Gil (1983), Perales (1994) y Baldaia (2006), quienes proponen dentro del modelo de transmisión recepción el Trabajo Práctico como complemento de la enseñanza teórica, que es transmitida por el docente, y que gracias a él podrá ser confirmada la temática trabajada. Según Baldaia (2006) bajo este modelo, el trabajo práctico asume un papel marcadamente demostrativo, en donde los conocimientos teóricos se confirman a través de actividades prácticas. Esta concepción implica entonces una Trabajo Práctico cuya enseñanza está centrada en la exposición que realiza el profesor, en donde los alumnos redescubren conceptos y hechos y su participación se reduce a la toma de apuntes (Rodrigo et al, 1999).

En cuanto a las implicaciones en la formación docente, cabe resaltar que uno de los aspectos que genera mayor controversia está relacionado con las relaciones entre la teoría y la práctica, en general ambos elementos han sido considerados como dimensiones separadas pero siempre atribuyéndole superioridad a la teoría sobre la práctica. De este modo, es importante que en la formación docente se reflexione sobre la forma en que se aborda la teoría y la práctica, partiendo de la idea de tratarlas como campos mutuamente constituyentes y relacionados. Asimismo la relación entre teoría y práctica no supone que la teoría implique la práctica, se derive de ella o la refleje, sino que la teoría transforma la práctica y esta a su vez puede transformar la teoría.

\section{CONCLUSIONES}

Los programas de formación de docentes de ciencias naturales que hemos mostrado aquí presentan una gran similitud con relación a la construcción de concepciones sobre enseñanza y aprendizaje de la Biología desde los contextos argentinos, españoles y colombianos, en donde se está favoreciendo el enriquecimiento de las ideas de los futuros docentes sobre enseñanza, aprendizaje, evaluación, finalidades de enseñanza, entre otros, particularmente desde la configuración de concepciones que se acercan a perspectivas más de corte constructivista que permite reconocer enseñanzas y aprendizajes que tengan más en cuenta los contextos y las particularidades de cada escuela.

Es importante favorecer procesos formativos en los cuales los estudiantes futuros maestros pongan en evidencia sus concepciones, pero además a través discusiones puedan detectarlas, modificarlas y re plantearlas en aras de una enseñanza-aprendizaje mucho más compleja y enriquecida desde perspectivas constructivistas, de allí la importancia de la inclusión de 
Memorias del VIII Encuentro Nacional de Experiencias en Enseñanza de la Biología y la Educación Ambiental. III Congreso Nacional de Investigación en Enseñanza de la Biología.

espacios de práctica pedagógica que permitan complejizar las concepciones de los futuros maestros, y que a través de diversas actividades les permitan reestructuras sus ideas.

\section{REFERENCIAS BIBLIOGRÁFICAS}

- Alarcón, Y \& Piñeros, I (1989). Las salidas de campo como un recurso pedagógico. Modelo de una salida. Tesis para optar al título de Licenciado en Biología y Química. Universidad de la Salle. Bogotá.

- Amórtegui, E; Gutiérrez, A; Medellín, F (2009). Las prácticas de campo en la construcción del conocimiento profesional de futuros profesores de Biología. En Biografía, escritos sobre la biología y su enseñanza. 2 (1).En: http://www.pedagogica.edu.co/revistas/ojs/index.php/biografia/article/viewFile/160/129.

- Amórtegui, E (2011). Concepciones sobre prácticas de campo y su relación con el conocimiento profesional del profesor, de futuros docentes de biología de la Universidad Pedagógica Nacional. Tesis para optar al título de Magíster en Educación. Bogotá: Universidad Pedagógica Nacional.

- Amórtegui, E Y Correa, M (2012). Las Prácticas de Campo Planificadas en el Proyecto Curricular de Licenciatura en Biología de la Universidad Pedagógica Nacional. Caracterización desde la perspectiva del Conocimiento Profesional del Profesor de Biología. Bogotá: Universidad Pedagógica Nacional y Fundación Francisca Radke.

- Amórtegui Elías y Valbuena Ussa Edgar (2010). Prácticas de campo en la enseñanza de la Biología y la formación docente: una revisión de antecedentes. IX Jornadas nacionales y IV Congreso internacional de enseñanza de la Biología. San Miguel de Tucumán 9 de octubre.

- Caamaño, A (2003). Los Trabajos Prácticos en Ciencias. En Jiménez (Coord) Enseñar Ciencias. Ed. Grao. Pp. 95-118.

- Dourado, L (2006). Concepçoes e práticas dos profesores de Ciencias Naturais Relativas à implementaçao Integrada do Trabalho Laboratorial e do Trabalho de Campo. Revista Electrónica de Enseñanza de las Ciencias. 5 (1). Pp. 192-212.

- Porlán, R. Y Rivero, A. (1998). El conocimiento de los profesores: una propuesta formativa en el área de ciencias. Sevilla: Díada.

- Puentes, M. y Valbuena, E. (2011). Sistema de categorías para análisis didáctico de trabajos prácticos en la enseñanza de la Biología. Bio-grafía. Escritos sobre la Biología y su Enseñanza. (en prensa).

- Rodrigo, M; Morcillo, J; Borges, R; Calvo, M; Cordeiro, N; García, F; Raviolo, A (1999) Concepciones sobre el Trabajo Práctico de campo (TPc): una aproximación al pensamiento de los futuros profesores. Revista Complutense de Madrid. 10 (2), 261285.

- Tardif, M. (2004). Los saberes del docente y su desarrollo profesional. Traducción de Pablo Manzano. Madrid: Narcea.

- Valbuena, E. (2007). El Conocimiento Didáctico del Contenido Biológico. Estudio de las concepciones disciplinares y didácticas de futuros docentes de la Universidad Pedagógica Nacional (Colombia). Tesis para optar al título de Doctor en Didáctica de las Ciencias Experimentales. Universidad Complutense de Madrid. 